\title{
Solving the Fractional Rosenau-Hyman Equation via Variational Iteration Method and Homotopy Perturbation Method
}

\author{
R. Yulita Molliq ${ }^{1}$ and M. S. M. Noorani ${ }^{2}$ \\ ${ }^{1}$ Department of Mathematics, Faculty of Mathematics and Natural Science, Universitas Negeri Medan \\ UNIMED 20221, Medan, Sumatera Utara, Indonesia \\ ${ }^{2}$ School of Mathematical Sciences, Faculty of Science and Technology, Universiti Kebangsaan Malaysia \\ UKM, 43600 Bangi, Selangor, Malaysia
}

Correspondence should be addressed to R. Yulita Molliq, yulitamolliq@yahoo.com

Received 31 May 2012; Accepted 8 November 2012

Academic Editor: Shaher Momani

Copyright (C) 2012 R. Yulita Molliq and M. S. M. Noorani. This is an open access article distributed under the Creative Commons Attribution License, which permits unrestricted use, distribution, and reproduction in any medium, provided the original work is properly cited.

\begin{abstract}
In this study, fractional Rosenau-Hynam equations is considered. We implement relatively new analytical techniques, the variational iteration method and the homotopy perturbation method, for solving this equation. The fractional derivatives are described in the Caputo sense. The two methods in applied mathematics can be used as alternative methods for obtaining analytic and approximate solutions for fractional Rosenau-Hynam equations. In these schemes, the solution takes the form of a convergent series with easily computable components. The present methods perform extremely well in terms of efficiency and simplicity.
\end{abstract}

\section{Introduction}

Recent advances of fractional differential equations are stimulated by new examples of applications in fluid mechanics, viscoelasticity, mathematical biology, electrochemistry, and physics. For example, the nonlinear oscillation of earthquake can be modeled with fractional derivatives [1], and the fluid-dynamic traffic model with fractional derivatives [2] can eliminate the deficiency arising from the assumption of continuum traffic flow. Based on experimental data fractional partial differential equations for seepage flow in porous media are suggested in [3], and differential equations with fractional order have recently proved to be valuable tools to the modeling of many physical phenomena [4]. Fractional partial differential equations also have studied and successfully solved such as the space-time fractional diffusion-wave equation [5-7], the fractional advection-dispersion equation [8, 9], 
the fractional $\mathrm{KdV}$ equation [10], and the linear inhomogeneous fractional partial differential equations [11].

Most nonlinear differential equations are usually arising from mathematical modeling of many physical systems. In most cases, it is very difficult to achieve analytic solutions of these equations. Perturbation techniques are widely used in science and engineering to handle nonlinear problems and do great contribution to help us understand many nonlinear phenomena. However, perturbation techniques are based on the existence of small/large parameter. Therefore, these techniques are not valid for strongly nonlinear problems.

The homotopy perturbation method (HPM) is the new approach for finding the approximate analytical solution of linear and nonlinear problems. The method was first proposed by He $[12,13]$ and was successfully applied to solve nonlinear wave equation by He [14-16]. The convergence of Homotopy perturbation series to the exact solution is considered in [17]. Similarly, applying the variational iteration method, created by $\mathrm{He}[3,18,19]$, consists in constructing the appropriate correction functional connected with the considered equation. The correction functional contains a Lagrange multiplier, the determination of which leads to a recurrence formula. Convergence of the VIM method is discussed by Tatari and Dehghan in [20]. Both of the methods examined have found application in determining the approximate solutions of different technical problems [21]. Adaptation of the VIM method for solving fractional heat-wave-like equation and fractional Zakharov-Kuznetsov equation were discussed by Yulita and colleagues in [22, 23]. Whereas, Chun [24] obtained the numerical solution of heat conduction problem by VIM. Recently, the application of the VIM method for solving kuramoto and Sivashinsky equations was presented by Porshokouhi and Ghanbari in [25]. For the application of HPM, this method used for solving fractional vibration equation [26] and partial differential equations of fractional order in finite domains [27].

In the present paper, VIM and HPM will be applied for solving fractional Rosenau-Hay nam equation which written as

$$
D_{t}^{\alpha} u=u D_{x x x}(u)+u D_{x}(u)+3 D_{x}(u) D_{x x}(u), \quad t>0,
$$

subject to the initial condition

$$
u(x, 0)=-\frac{8}{3} c \cos ^{2}\left(\frac{x}{4}\right)
$$

where $u=u(x, t), \alpha$ is a parameter describing the order of the fractional derivative $(0<\alpha \leq$ $1), t$ is the time, and $x$ is the spatial coordinate. Fractional RH equation when $\alpha=1.0$ has appeared in the study of the formation of patterns in liquid drops [28].

\section{Basic Definitions}

Fractional calculus unifies and generalizes the notions of integer-order differentiation and $n$ fold integration $[4,29]$. We give some basic definitions and properties of fractional calculus theory which will be used in this paper: 
Definition 2.1. A real function $f(x), x>0$ is said to be in the space $\mathcal{C}_{\mu}, \mu \in \mathbf{R}$ if there exists a real number $p(>\mu)$, such that $f(x)=x^{p} f_{1}(x)$, where $f_{1}(x) \in \mathcal{C}[0, \infty)$, and it is said to be in the space $\mathcal{C}_{\mu}^{m}$ if and only if $f^{(m)} \in \mathcal{C}_{m}, m \in \mathbf{N}$.

The Riemann-Liouville fractional integral operator is defined as follows.

Definition 2.2. The Riemann-Liouville fractional integral operator of order $\alpha \geq 0$, of a function $f \in C_{\mu}, \mu \geq-1$, is defined as

$$
\begin{gathered}
J^{\alpha} f(x)=\frac{1}{\Gamma(\alpha)} \int_{0}^{x}(x-t)^{\alpha-1} f(t) \mathrm{d} t, \quad \alpha>0, x>0, \\
J^{0} f(x)=f(x)
\end{gathered}
$$

In this paper only real and positive values of $\alpha$ will be considered.

Properties of the operator $J^{\alpha}$ can be found in [29] and we mention only the following: for $f \in C_{\mu}, \mu \geq-1, \alpha, \beta \geq 0$, and $\gamma \geq-1$ :

(1) $J^{\alpha} J^{\beta} f(x)=J^{\alpha+\beta} f(x)$,

(2) $J^{\alpha} J^{\beta} f(x)=J^{\beta} J^{\alpha} f(x)$,

(3) $J^{\alpha} x^{\gamma}=(\Gamma(\gamma+1) / \Gamma(\alpha+\gamma+1)) x^{\alpha+\gamma}$.

The Reimann-Liouville derivative has certain disadvantages when trying to model realworld phenomena with FDEs. Therefore, we will introduce a modified fractional differential operator $D_{*}^{\alpha}$ proposed by Caputo in his work on the theory of viscoelasticity [30].

Definition 2.3. The fractional derivative of $f(x)$ in Caputo sense is defined as

$$
\begin{array}{r}
D_{*}^{\alpha} f(x)=J^{m-\alpha} D_{*}^{m} f(x)=\frac{1}{\Gamma(m)} \int_{0}^{x}(x-s)^{m-\alpha-1} f^{(m)}(s) \mathrm{d} s, \\
\quad \text { for } m-1<\alpha \leq m, m \in \mathcal{N}, x>0, f \in C_{-1}^{m} .
\end{array}
$$

In addition, we also need the following property.

Lemma 2.4. If $m-1<\alpha \leq m, m \in \mathcal{N}$ and $f \in C_{\mu}^{m}, \mu \geq-1$, then

$$
\begin{gathered}
D_{*}^{\alpha} J^{\alpha} f(x)=f(x), \\
J^{\alpha} D_{*}^{\alpha} f(x)=f(x)-\sum_{i=0}^{m-1} f^{(i)}\left(0^{+}\right) \frac{x^{i}}{i !}, \quad x>0 .
\end{gathered}
$$

The Caputo differential derivative is considered here because the initial and boundary conditions can be included in the formulation of the problems [4]. The fractional derivative is taken in the Caputo sense as follows. 
Definition 2.5. For $m$ to be the smallest integer that exceeds $\alpha$, the Caputo fractional derivative operator of order $\alpha>0$ is defined as

$$
D_{t}^{\alpha} u(x, t)= \begin{cases}\frac{1}{\Gamma(m-\alpha)} \int_{0}^{t}(t-s)^{m-\alpha-1\left(\partial^{m} u(x, s) / \partial s^{m}\right)} \mathrm{d} s, & \text { for } m-1<\alpha \leq m, \\ \frac{\partial^{m} u(x, t)}{\partial t^{m}}, & \text { for } \alpha=m \in \mathbf{N} .\end{cases}
$$

For more information on the mathematical properties of fractional derivatives and integrals, one can consult $[4,29]$.

\section{Basic Idea of He's Variational Iteration Method}

To clarify the basic ideas of VIM, we consider the following differential equation:

$$
D_{* t}^{\alpha}(u)=f\left(u, u_{x}, u_{x x}\right)+g(x, t), \quad m-1<\alpha<m,
$$

where $u=u(x, t), D_{* t}^{\alpha}=\partial^{\alpha} / \partial t^{\alpha}$ is the Caputo fractional derivative of order $\alpha, m \in N, f$ is a nonlinear function, and $g$ is the source function. According to VIM, we can write down a correction functional as follows:

$$
u_{n+1}(x, t)=u_{n}(x, t)+\int_{0}^{t} \lambda(s)\left[\frac{\partial^{m}}{\partial s^{m}}\left(u_{n}\right)-f\left(\tilde{u}_{n}, \tilde{u}_{n, x}, \tilde{u}_{n, x x}\right)-g(x, s)\right] \mathrm{d} s,
$$

where $\lambda(s)$ is a general Lagrangian multiplier which can be optimally identified via the variational theory [31]. $\tilde{u}_{n}$ is considered as a restricted variation [32], that is, $\delta \tilde{u}_{n}=0$ and the subscript $n$ indicates the $n$th approximation. We have

$$
\delta u_{n+1}=\delta u_{n}+\delta \int_{0}^{t} \lambda(s)\left[\frac{\partial^{m}}{\partial s^{m}}\left(u_{n}\right)-f\left(\tilde{u}_{n}, \tilde{u}_{n, x}, \tilde{u}_{n, x x}\right)-g(x, s)\right] \mathrm{d} s
$$

where $\tilde{u}_{n}$ is considered as restricted variations, that is, $\delta \tilde{u}_{n}=0$. For $m=1$, we have

$$
\begin{aligned}
& \delta u_{n+1}=\delta u_{n}+\delta \int_{0}^{t} \lambda(s)\left[\frac{\partial}{\partial s} u_{n}\right] \mathrm{d} s \\
& \delta u_{n+1}=\left[1-\lambda^{\prime}(t)\right] \delta u_{n}+\delta \lambda(t) \frac{\partial}{\partial t} u_{n}+\int_{0}^{1} \delta \lambda^{\prime \prime}(s) u_{n} \mathrm{~d} s .
\end{aligned}
$$

Thus, we obtain the following stationary conditions:

$$
\begin{gathered}
\delta u_{n}(t):\left.\left[1-\lambda^{\prime}(t)\right]\right|_{s=t}=0, \\
\delta u_{n}^{\prime}(t):\left.\lambda(t)\right|_{s=t}=0, \\
\delta u_{n}(s): \lambda^{\prime \prime}(t)=0
\end{gathered}
$$


Solving this system of equations yields

$$
\lambda(s)=-1 .
$$

Furthermore, substituting (3.6) to (3.2), the iteration formula of VIM can be written as follows:

$$
u_{n+1}=u_{n}-\int_{0}^{t}\left[\frac{\partial^{m}}{\partial s^{m}}\left(u_{n}\right)-f\left(u_{n}, u_{n, x}, u_{n, x x}\right)-g(x, s)\right] \mathrm{d} s .
$$

In this case, we begin with the initial approximation:

$$
u_{0}(x, t)=h(x)
$$

The correction functional (3.8) will give several approximations, and therefore the exact solution is obtained as

$$
u(x, t)=\lim _{n \rightarrow \infty} u_{n}(x, t)
$$

\section{Basic Idea of Homotopy Perturbation Method}

Consider the following nonlinear differential equation:

$$
A(u)-f(r)=0,
$$

with boundary conditions

$$
B\left(u, \frac{\partial u}{\partial n}\right)=0, \quad r \in \Gamma
$$

where $A$ is a general differential operator, $B$ is a boundary operator, $f(r)$ is a known analytic function, and $\Gamma$ is the boundary of the domain $\Omega$.

The operator $A$ can, generally speaking, be divided into two parts, $L$ and $N$, where $L$ is linear and $N$ is nonlinear, therefore (4.1) can be written as

$$
L(u)+N(u)-f(r)=0
$$

By using homotopy technique, one can construct a homotopy $v(r, p): \Omega \times[0,1] \rightarrow \mathfrak{R}$ which satisfies

$$
H(v, p)=(1-p)\left[L(v)-L\left(u_{0}\right)\right]+p[A(v)-f(r)]=0
$$

or

$$
H(v, p)=L(v)-L\left(u_{0}\right)+p L\left(u_{0}\right)+p[N(v)-f(r)]=0,
$$


where $p \in[0,1]$ is an embedding parameter, and $u_{0}$ is the initial approximation of (4.1) which satisfies the boundary conditions. Clearly, we have

$$
H(v, 0)=L(v)-L(u 0)=0,
$$

or

$$
H(v, 1)=A(v)-f(r)=0,
$$

the changing process of $p$ from zero to unity is just that of $v(r, p)$ changing from $u_{0}(r)$ to $u(r)$. This is called deformation, and also, $L(v)-L\left(u_{0}\right)$ and $A(v)-f(r)$ are called homotopic in topology. If the embedding parameter $p(0 \leq p \leq 1)$ is considered as a small parameter, applying the classical perturbation technique, we can assume that the solution of (4.3) and (4.4) can be given as a power series in $p$, that is,

$$
v=v_{0}+p v_{1}+p^{2} v_{2}+\cdots
$$

and setting $p=1$ results in the approximate solution of (4.1) as

$$
u=\lim _{p \rightarrow 1} v=v_{0}+v_{1}+v_{2}+\cdots
$$

\section{Application of HPM and VIM Methods}

We consider the application of VIM to fractional Rosenau-Hynam equations which is rewritten as follows:

$$
D_{t}^{\alpha} u=u D_{x x x}(u)+u D_{x}(u)+3 D_{x}(u) D_{x x}(u),
$$

where $u=u(x, t)$ with the initial conditions of

$$
u(x, 0)=-\frac{8}{3} c \cos ^{2}\left(\frac{x}{4}\right)
$$

\subsection{VIM Implement for Fractional Rosenau-Hynam Equation}

According to the formula (3.8), the iteration formula for (4.9) is given by

$$
u_{n+1}=u_{n}-\int_{0}^{t}\left[D_{s}^{\alpha}\left(u_{n}\right)-u_{n} D_{x x x}\left(u_{n}\right)-u_{n} D_{x}\left(u_{n}\right)-3 D_{x}\left(u_{n}\right) D_{x x}\left(u_{n}\right)\right] \mathrm{d} s .
$$




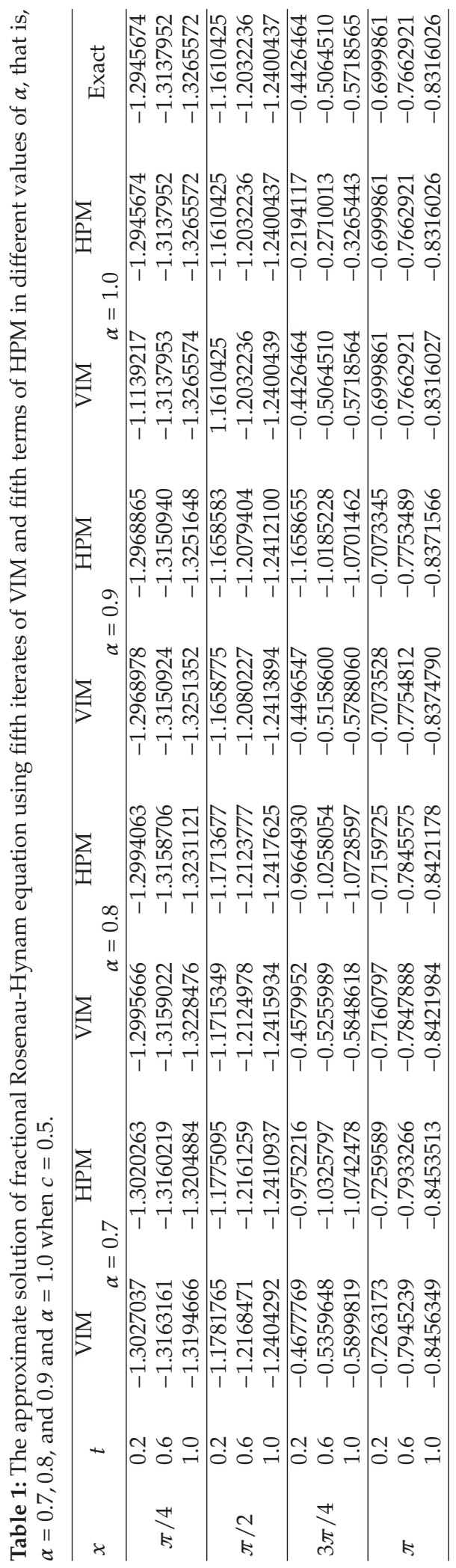




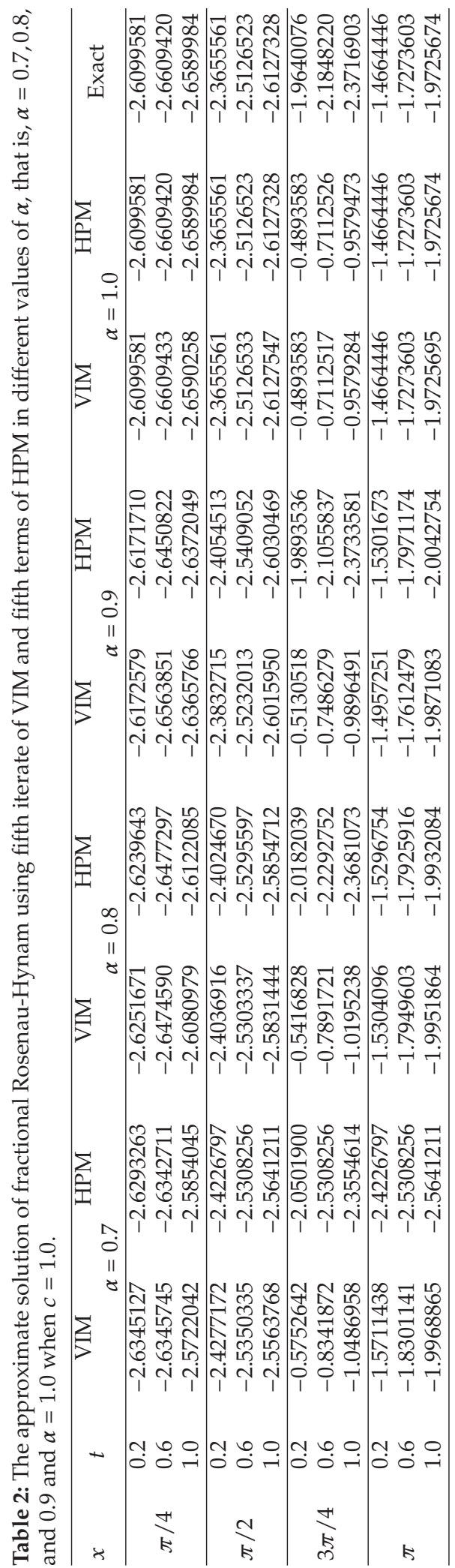


Table 3: Errors of the approximate solution of fractional Rosenau-Hynam equation using using fifth term of HPM and fifth iterate of VIM when $\alpha=1.0$ and $c=0.5$.

\begin{tabular}{lcccccc}
\hline \multirow{2}{*}{$t$} & \multicolumn{2}{c}{$x=\pi / 2$} & \multicolumn{2}{c}{$x=\pi$} & \multicolumn{2}{c}{$x=3 \pi / 2$} \\
& VIM & HPM & VIM & HPM & VIM & HPM \\
\hline 0.1 & $1.0000 E-11$ & $1.0000 E-11$ & $5.0000 E-10$ & $1.0000 E-11$ & $2.0000 E-10$ & $1.0000 E-11$ \\
0.2 & $1.0000 E-11$ & $1.2173 E-09$ & $5.0000 E-10$ & $1.7360 E-09$ & $3.0000 E-10$ & $1.2378 E-09$ \\
0.3 & $1.0000 E-11$ & $9.2044 E-09$ & $5.0000 E-10$ & $1.3182 E-08$ & $3.0000 E-10$ & $9.4375 E-09$ \\
0.4 & $1.0000 E-10$ & $3.8620 E-08$ & $1.0000 E-10$ & $5.5542 E-08$ & $9.0000 E-10$ & $3.9929 E-08$ \\
0.5 & $3.0000 E-10$ & $1.1734 E-07$ & $4.0000 E-10$ & $1.6948 E-07$ & $1.9000 E-09$ & $1.2233 E-07$ \\
0.6 & $1.0000 E-09$ & $2.9070 E-07$ & $7.0000 E-10$ & $4.2165 E-07$ & $6.1000 E-09$ & $3.0561 E-07$ \\
0.7 & $2.6000 E-09$ & $6.2550 E-07$ & $1.2000 E-09$ & $9.1117 E-07$ & $1.8300 E-08$ & $6.6309 E-07$ \\
0.8 & $5.4000 E-09$ & $1.2140 E-06$ & $2.1000 E-09$ & $1.7761 E-06$ & $3.9300 E-08$ & $1.2978 E-06$ \\
0.9 & $1.1110 E-07$ & $2.1777 E-06$ & $4.0000 E-09$ & $3.1998 E-06$ & $8.2200 E-08$ & $2.3474 E-06$ \\
1.0 & $2.1110 E-07$ & $3.6709 E-06$ & $8.6000 E-09$ & $5.4173 E-06$ & $1.5230 E-07$ & $3.9903 E-06$ \\
\hline
\end{tabular}

Table 4: Errors of the approximate solution of fractional Rosenau-Hynam equation using fifth term of HPM and fifth iterate of VIM when $\alpha=1.0$ and $c=0.5$.

\begin{tabular}{lcccccc}
\hline \multirow{t}{t}{} & \multicolumn{2}{c}{$x=\pi / 2$} & \multicolumn{2}{c}{$x=\pi$} & \multicolumn{2}{c}{$x=3 \pi / 2$} \\
& VIM & HPM & VIM & HPM & VIM & ADM \\
\hline 0.1 & $1.0000 E-11$ & $2.4346 E-09$ & $5.0000 E-10$ & $3.4720 E-09$ & $2.0000 E-10$ & $2.4756 E-09$ \\
0.2 & $1.0000 E-11$ & $7.7240 E-08$ & $5.0000 E-10$ & $1.1109 E-07$ & $3.0000 E-10$ & $7.9858 E-08$ \\
0.3 & $1.0000 E-11$ & $5.8140 E-07$ & $5.0000 E-10$ & $8.4330 E-07$ & $3.0000 E-10$ & $6.1121 E-07$ \\
0.4 & $1.0000 E-10$ & $2.4280 E-06$ & $1.0000 E-10$ & $3.5522 E-06$ & $9.0000 E-10$ & $2.5955 E-06$ \\
0.5 & $3.0000 E-10$ & $7.3419 E-06$ & $4.0000 E-10$ & $1.0835 E-05$ & $1.9000 E-09$ & $7.9805 E-06$ \\
0.6 & $1.0000 E-09$ & $1.8100 E-05$ & $7.0000 E-10$ & $2.6942 E-05$ & $6.1000 E-09$ & $2.0004 E-05$ \\
0.7 & $2.6000 E-09$ & $3.8743 E-05$ & $1.2000 E-09$ & $5.8188 E-05$ & $1.8300 E-08$ & $4.3547 E-05$ \\
0.8 & $5.4000 E-09$ & $7.4800 E-05$ & $2.1000 E-09$ & $1.1335 E-04$ & $3.9300 E-08$ & $8.5496 E-05$ \\
0.9 & $1.1110 E-07$ & $1.3340 E-04$ & $4.0000 E-09$ & $2.0405 E-04$ & $8.2200 E-08$ & $1.5512 E-04$ \\
1.0 & $2.1110 E-07$ & $2.2370 E-04$ & $8.6000 E-09$ & $3.4516 E-04$ & $1.5230 E-07$ & $2.6444 E-04$ \\
\hline
\end{tabular}

The iteration starts with an initial approximation which is initial condition in (5.2). Furthermore, using the iteration formula in (5.3), we can directly obtain other components as

$$
\begin{aligned}
u_{1}(x, t)= & -\frac{2}{3} c^{2} \sin \left(\frac{x}{2}\right) t-\frac{4}{3} c\left[1+\cos \left(\frac{x}{2}\right)\right], \\
u_{2}(x, t)= & -\frac{4}{3} c^{2} \sin \left(\frac{x}{2}\right)+\left[\frac{1}{6} c^{2}+\frac{4}{3} c\right]\left(1+\cos \left(\frac{x}{2}\right)\right) \\
& -\frac{1}{6} c^{3} t^{2}+\frac{2}{3} c^{2} \sin \left(\frac{x}{2}\right) \frac{t^{(2-\alpha)}}{\Gamma(3-\alpha)}, \\
u_{3}(x, t)= & \frac{7}{6} c^{3} t^{3} \cos \left(\frac{x}{2}\right)-\frac{96}{36} \cos ^{2}\left(\frac{x}{4}\right)-\frac{48}{36} c^{2} t \sin \left(\frac{x}{2}\right)-\frac{2}{3} \cos \left(\frac{x}{2}\right) \frac{t^{3-\alpha}}{\Gamma(4-\alpha)} \\
& +(6-2 \alpha) c^{2} \sin \left(\frac{x}{2}\right) \frac{t^{2-\alpha}}{\Gamma(4-\alpha)}-\frac{2}{3} c^{2} \sin \left(\frac{x}{2}\right) \frac{t^{3-2 \alpha}}{\Gamma(4-2 \alpha)},
\end{aligned}
$$

and so on. 


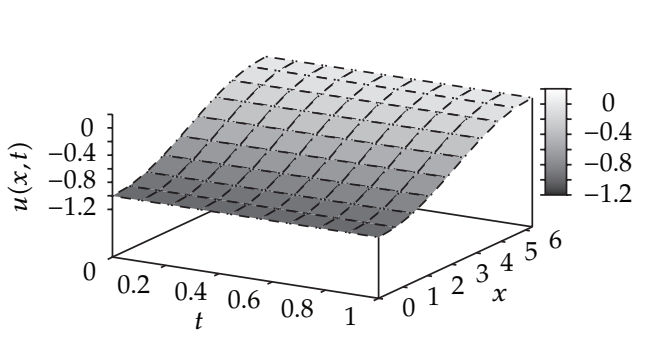

(a)

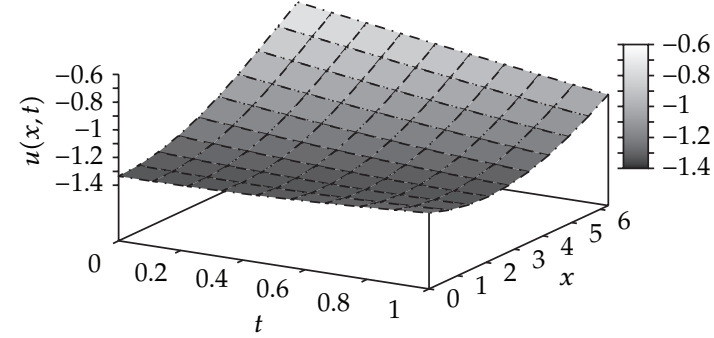

(b)

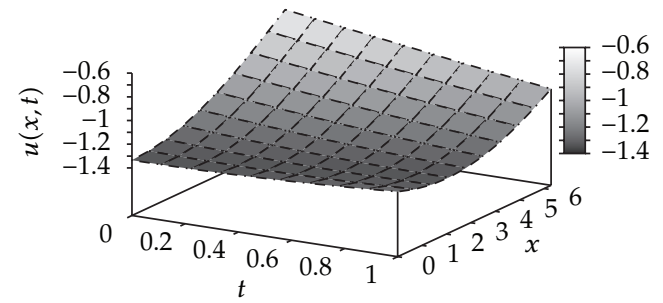

(c)

Figure 1: The approximate solution of fractional Rosenau-Hynam equation when $\alpha=1.0$ and $c=0.5$ using such method: (a) HPM, (b) VIM and (c) Exact.

The exact solution of this equation is given by [33]

$$
u(x, t)=-\frac{8}{3} c \cos ^{2}\left(\frac{1}{4}(x-c t)\right), \quad|x-c t| \leq 2 \pi,
$$

where $c$ is arbitrary constant [28].

\section{Analysis of the Homotopy Perturbation Method (HPM)}

Now applying the classical perturbation technique for solving (5.1) with initial condition in (5.2). To solve (5.1) by the homotopy perturbation method, we construct the following homotopy:

$$
\begin{gathered}
(1-p) D_{t}^{\alpha} u(x, t)+p\left[D_{t}^{\alpha} u(x, t)-\left(u(x, t) D_{x x x} u(x, t)+u(x, t) D_{x} u(x, t)\right.\right. \\
\left.\left.+3 D_{x} u(x, t) D_{x x} u(x, t)\right)\right]=0
\end{gathered}
$$

or

$$
D_{t}^{\alpha} u(x, t)=p\left[u(x, t) D_{x x x} u(x, t)+u(x, t) D_{x} u(x, t)+3 D_{x} u(x, t) D_{x x} u(x, t)\right]
$$

where $p \in[0,1]$ is an embedding parameter. If $p=0$, then (6.2) becomes a linear equation,

$$
D_{t}^{\alpha} u(x, t)=0 .
$$




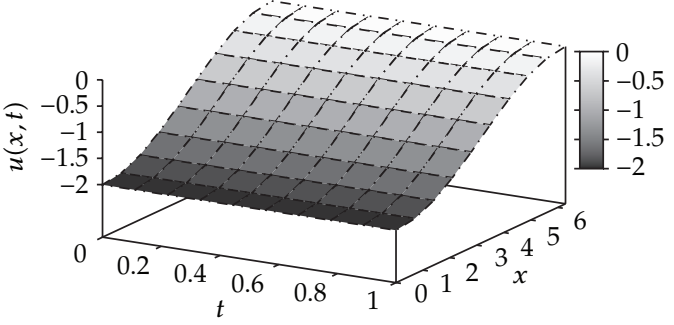

(a)

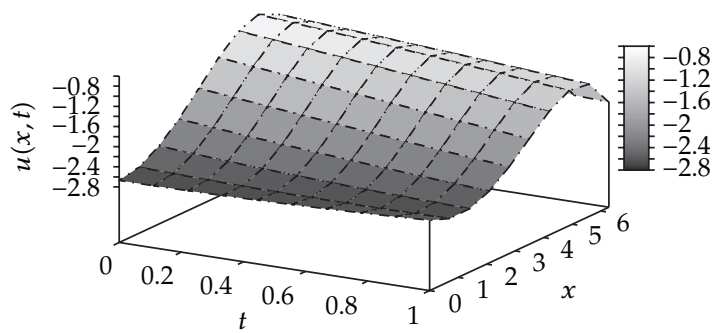

(b)

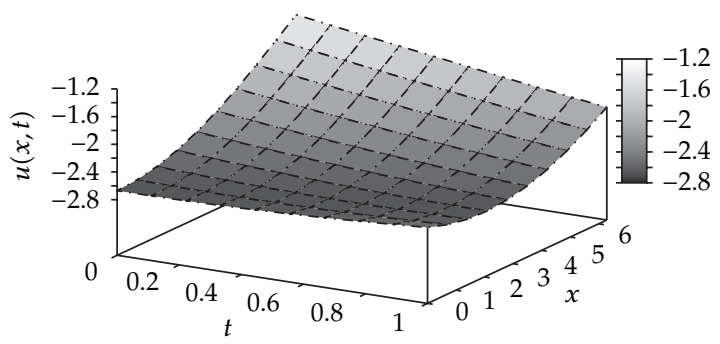

(c)

Figure 2: The approximate solution of fractional Rosenau-Hynam equation when $\alpha=1.0$ and $c=1.0$ using such method: (a) HPM, (b) VIM, and (c) Exact.

And when $p=1$, then (6.2) turns out to be (5.1). Assume the solution of (6.2) to be in the form

$$
u(x, t)=v_{0}+p v_{1}+p^{2} v_{2}+p^{3} v_{3}+\cdots
$$

Substituting (6.4) into (6.2) and equating the terms with identical powers of $p$, we obtain the following set of linear differential equations:

$$
\begin{aligned}
& p^{0}: D_{t}^{\alpha} u_{0}=0 \\
& p^{1}: D_{t}^{\alpha} u_{1}=v_{0} \frac{\partial v_{0}}{\partial x}+3 \frac{\partial v_{0}}{\partial x} \frac{\partial^{2} v_{0}}{\partial x^{2}}+v_{0} \frac{\partial^{3} v_{0}}{\partial x^{3}} \\
& p^{2}: D_{t}^{\alpha} u_{2}=v_{1} \frac{\partial v_{0}}{\partial x}+v_{0} \frac{\partial v_{1}}{\partial x}+3 \frac{\partial v_{1}}{\partial x} \frac{\partial^{2} v_{0}}{\partial x^{2}}+3 \frac{\partial v_{0}}{\partial x} \frac{\partial^{2} v_{1}}{\partial x^{2}}+v_{1} \frac{\partial^{3} v_{0}}{\partial x^{3}}+v_{0} \frac{\partial^{3} v_{1}}{\partial x^{3}}
\end{aligned}
$$

and so on. Equations (6.6) and (6.7) can be solved by applying the operator $J^{\alpha}$, which is the inverse of the operator $D^{\alpha}$ and then by simple computation, Thus, the solution reads as follows

$$
\begin{aligned}
& v_{1}(x, t)=-\frac{2 c^{2} t^{\alpha} \sin (x / 4)}{3 \Gamma(1+\alpha)} \\
& v_{2}(x, t)=-\frac{c^{3}}{3 \pi} t^{2 \alpha} \cos \left(\frac{x}{2}\right) \Gamma(-2 \alpha) \sin 1 \pi \alpha
\end{aligned}
$$




$$
\begin{aligned}
& v_{3}(x, t)=-\frac{c^{4} \pi t^{3 \alpha} \csc \pi \alpha \sin (x / 2)}{6 \Gamma(1-\alpha) \Gamma(\alpha) \Gamma(1+3 \alpha)} \\
& v_{4}(x, t)=-\frac{c^{5} \pi t^{4 \alpha} \cos (x / 2) \csc \alpha \pi}{12 \Gamma(1-\alpha) \Gamma(\alpha) \Gamma(1+4 \alpha)}
\end{aligned}
$$

and so on.

In this manner, the rest of the components of the homotopy perturbation series can be obtained. Finally, we approximate the analytical solutions of $u(x, t)$ by the truncated series

$$
u(x, t)=\lim _{N \rightarrow \infty} u_{5}(x, t),
$$

where $u_{5}(x, t)=\sum_{n=0}^{4} v_{k}(x, t)$.

\section{Numerical Results and Discussion}

Tables 1 and 2 show the approximate solutions for (5.1) obtained for different values of $\alpha$ using the decomposition method and the variational iteration method in different values of $c$, that is, $c=1$ and $c=0.5$, respectively. Tables 3 and 4 show the absolute error of (5.1) when $\alpha=1.0$ in different value of $c$, that is, $c=1.0$ and $c=0.5$, respectively. Figures 1 and 2 show the approximate solutions for (5.1) in different values of $c$ using the fifth iterates of VIM, the fifth terms of HPM when $\alpha=1$, and exact solution, respectively. From Tables 3 and 4 show that the approximate solution using the VIM is more accurate than the approximate solution obtained using the HPM. It is to be noted that only the fifth iterates of the variational iteration solution and only fifth terms of the homotopy perturbation series were used in evaluating.

\section{Conclusions}

The fundamental goal of this work has been to construct an approximate solution of nonlinear partial differential equations of fractional order. For computations and plots, the Mathematica and Maple packages were used. The goal has been achieved by using the variational iteration method (VIM) and the homotopy perturbation method (HPM). The methods were used in a direct way without using linearization or restrictive assumptions. There are four important points that were gotten. First, the VIM and the HPM provide the solutions in terms of convergent series with easily computable components. Second, the approximate solution in (5.1) using the VIM converges faster than the approximate solution using the HPM. Third, the variational iteration method handles nonlinear equations without any need for the socalled He's polynomials. Finally, the recent appearance of fractional differential equations as models in some fields of applied mathematics makes it necessary to investigate methods of solution for such equations (analytical and numerical) and we hope that this work is a step in this direction. 


\section{Acknowledgment}

The financial support received from UKM Grant ERGS/1/2011/STG/UKM/01/13 is gratefully acknowledged.

\section{References}

[1] J. H. He, "Semi-inverse method of establishing generalized variational principles for fluid mechanics with emphasis on turbomachinery aerodynamics," International Journal of Turbo and Jet Engines, vol. 14, no. 1, pp. 23-28, 1997.

[2] J. H. He, "Some applications of nonlinear fractional differential equations and their approximations," Bulletin of Science, Technology \& Society, vol. 15, no. 2, pp. 86-90, 1999.

[3] J. H. He, "Approximate analytical solution for seepage flow with fractional derivatives in porous media," Computer Methods in Applied Mechanics and Engineering, vol. 167, no. 1-2, pp. 57-68, 1998.

[4] I. Podlubny, Fractional Differential Equations, vol. 198 of Mathematics in Science and Engineering, Academic Press, San Diego, Calif, USA, 1999.

[5] K. Al-Khaled and S. Momani, "An approximate solution for a fractional diffusion-wave equation using the decomposition method," Applied Mathematics and Computation, vol. 165, no. 2, pp. 473-483, 2005.

[6] F. Mainardi, Y. Luchko, and G. Pagnini, "The fundamental solution of the space-time fractional diffusion equation," Fractional Calculus E Applied Analysis, vol. 4, no. 2, pp. 153-192, 2001.

[7] A. Hanyga, "Multidimensional solutions of time-fractional diffusion-wave equations," Proceedings of the Royal Society of London, Series A, vol. 458, no. 2020, pp. 933-957, 2002.

[8] F. Huang and F. Liu, "The time fractional diffusion equation and the advection-dispersion equation," The Australian \& New Zealand Industrial and Applied Mathematics Journal, vol. 46, no. 3, pp. 317-330, 2005.

[9] F. Huang and F. Liu, "The fundamental solution of the space-time fractional advection-dispersion equation," Journal of Applied Mathematics E Computing, vol. 18, no. 1-2, pp. 339-350, 2005.

[10] S. Momani, "An explicit and numerical solutions of the fractional KdV equation," Mathematics and Computers in Simulation, vol. 70, no. 2, pp. 110-118, 2005.

[11] L. Debnath and D. D. Bhatta, "Solutions to few linear fractional inhomogeneous partial differential equations in fluid mechanics," Fractional Calculus \& Applied Analysis, vol. 7, no. 1, pp. 21-36, 2004.

[12] J. H. He, "Homotopy perturbation technique," Computer Methods in Applied Mechanics and Engineering, vol. 178, no. 3-4, pp. 257-262, 1999.

[13] J. H. He, "A coupling method of a homotopy technique and a perturbation technique for non-linear problems," International Journal of Non-Linear Mechanics, vol. 35, no. 1, pp. 37-43, 2000.

[14] J. H. He, "Periodic solutions and bifurcations of delay-differential equations," Physics Letters A, vol. 347, no. 4-6, pp. 228-230, 2005.

[15] J. H. He, "Application of homotopy perturbation method to nonlinear wave equations," Chaos, Solitons and Fractals, vol. 26, no. 3, pp. 695-700, 2005.

[16] J. H. He, "Limit cycle and bifurcation of nonlinear problems," Chaos, Solitons and Fractals, vol. 26, no. 3, pp. 827-833, 2005.

[17] J. H. He, Non-perturbative methods for strongly nonlinear problems [Dissertation], de-Verlag im Internet GmbH, Berlin, Germany, 2006.

[18] J. H. He, "A new approach to nonlinear partial differential equations," Communications in Nonlinear Science and Numerical Simulation, vol. 2, no. 4, pp. 230-235, 1997.

[19] J. H. He, "Variational iteration method for delay differential equations," Communications in Nonlinear Science and Numerical Simulation, vol. 2, no. 4, pp. 235-236, 1997.

[20] M. Tatari and M. Dehghan, "On the convergence of He's variational iteration method," Journal of Computational and Applied Mathematics, vol. 207, no. 1, pp. 121-128, 2007.

[21] Z. Odibat and S. Momani, "Numerical methods for nonlinear partial differential equations of fractional order," Applied Mathematical Modelling, vol. 32, no. 1, pp. 28-39, 2008.

[22] R. Yulita Molliq, M. S. M. Noorani, and I. Hashim, "Variational iteration method for fractional heatand wave-like equations," Nonlinear Analysis: Real World Applications, vol. 10, no. 3, pp. 1854-1869, 2009. 
[23] R. Yulita Molliq, M. S. M. Noorani, I. Hashim, and R. R. Ahmad, “Approximate solutions of fractional Zakharov-Kuznetsov equations by VIM," Journal of Computational and Applied Mathematics, vol. 233, no. 2, pp. 103-108, 2009.

[24] C. Chun, "Variational iteration method for a reliable treatment of heat equations with ill-defined initial data," International Journal of Nonlinear Sciences and Numerical Simulation, vol. 9, no. 4, pp. 435 440, 2008.

[25] M. G. Porshokouhi and B. Ghanbari, "Application of He's variational iteration method for solution of the family of Kuramoto-Sivashinsky equations," Journal of King Saud University - Science, vol. 23, no. 4, pp. 407-411, 2011.

[26] S. T. Mohyud-Din and A. Yildirim, "An algorithm for solving the fractional vibration equation," Computational Mathematics and Modeling, vol. 23, no. 2, pp. 228-237, 2012.

[27] A. M. A. El-Sayed, A. Elsaid, I. L. El-Kalla, and D. Hammad, "A homotopy perturbation technique for solving partial differential equations of fractional order in finite domains," Applied Mathematics and Computation, vol. 218, no. 17, pp. 8329-8340, 2012.

[28] P. Rosenau and J. M. Hyman, "Compactons: solitons with finite wavelength," Physical Review Letters, vol. 70, no. 5, pp. 564-567, 1993.

[29] R. Gorenflo and F. Mainardi, "Fractional calculus: integral and differential equations of fractional order," in Fractals and Fractional Calculus, A. Carpinteri and F. Mainardi, Eds., Springer, New York, NY, USA, 1997.

[30] M. Caputo, "Linear models of dissipation whose Q is almost frequency independent II," Geophysical Journal of the Royal Astronomical Society, vol. 13, no. 5, pp. 529-539, 1967.

[31] M. Inokuti, H. Sekine, and T. Mura, "General use of the Lagrange multiplier in nonlinear mathematical physics," in Variational Method in the Mechanics of Solids, S. Nemat Nasser, Ed., pp. 156162, Pergamon Press, New York, NY, USA, 1978.

[32] B. A. Finlayson, The Method of Weighted Residuals and Variational Principles, Academic Press, New York, NY, USA, 1972.

[33] P. A. Clarkson, E. L. Mansfield, and T. J. Priestley, "Symmetries of a class of nonlinear third-order partial differential equations," Mathematical and Computer Modelling, vol. 25, no. 8-9, pp. 195-212, 1997. 


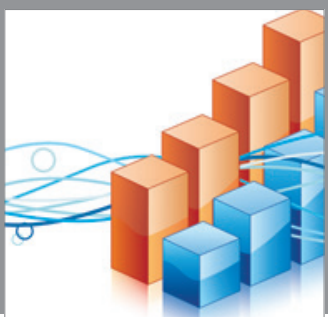

Advances in

Operations Research

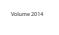

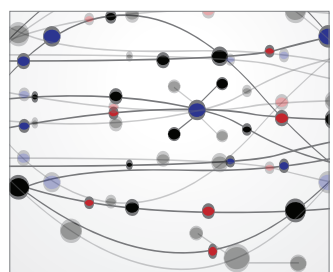

\section{The Scientific} World Journal
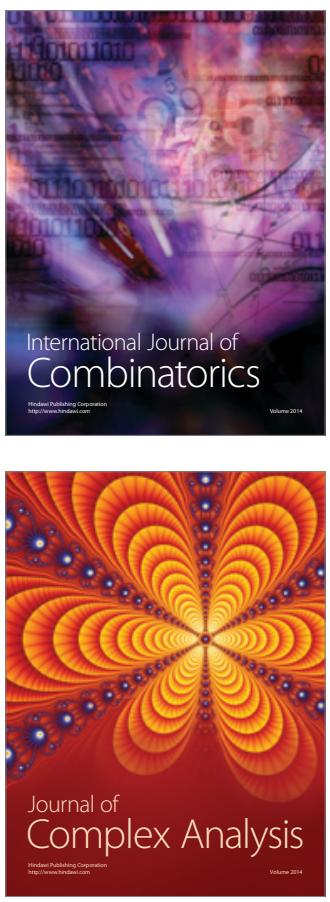

International Journal of

Mathematics and

Mathematical

Sciences
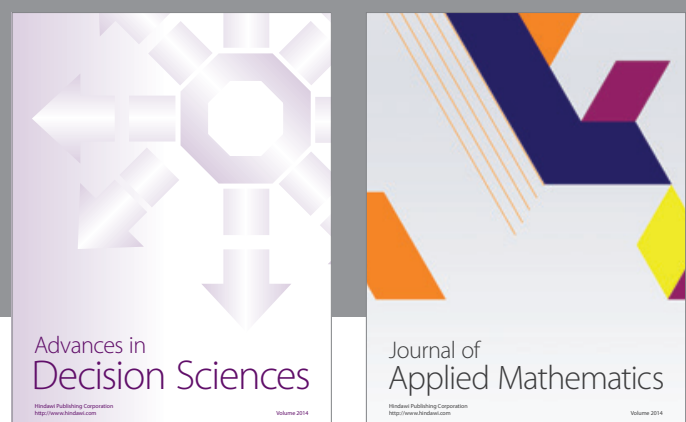

Journal of

Applied Mathematics
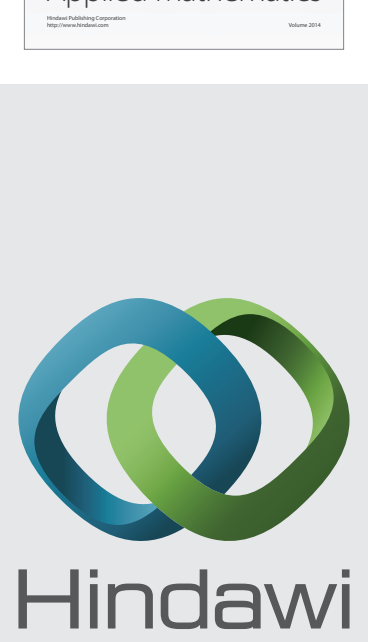

Submit your manuscripts at http://www.hindawi.com
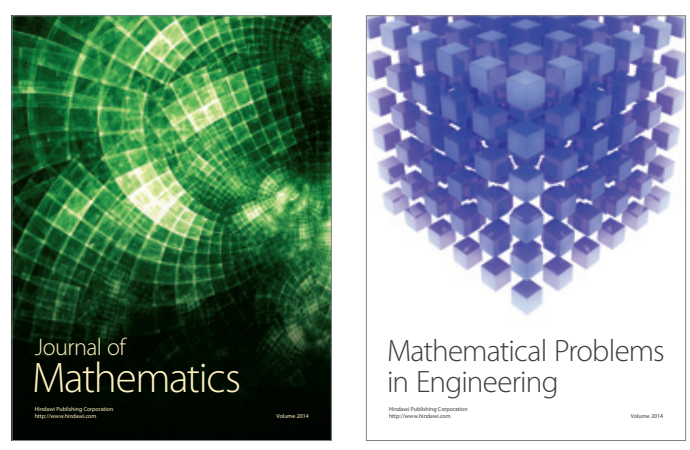

Mathematical Problems in Engineering
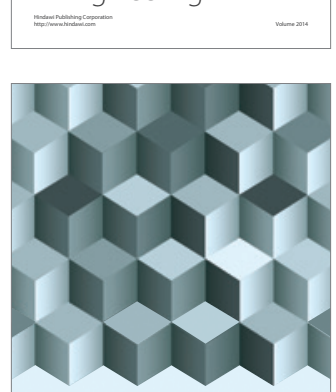

Journal of

Function Spaces
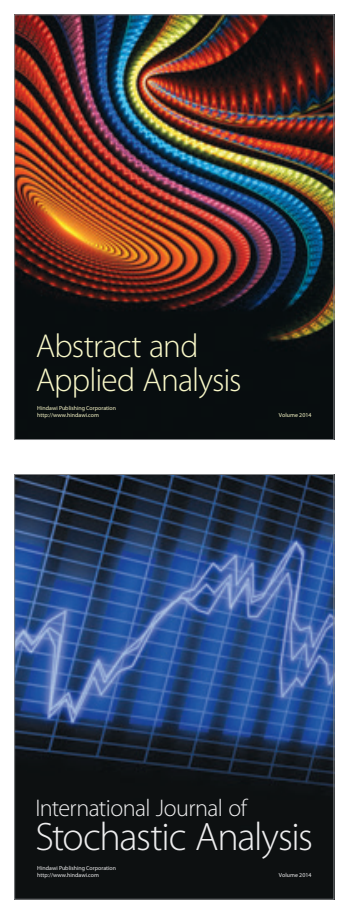

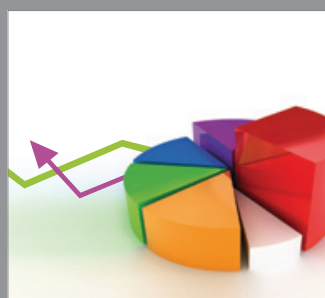

ournal of

Probability and Statistics

Promensencen
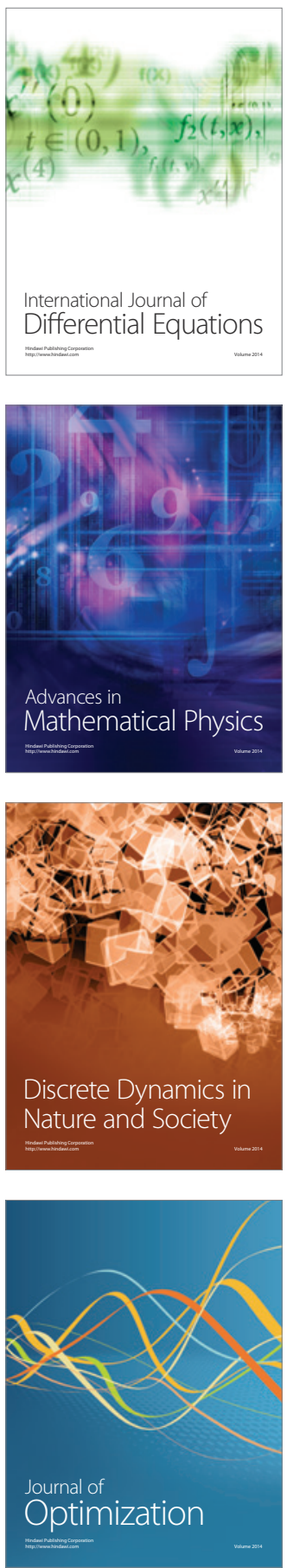